\title{
EXERCÍCIOS E ELETROESTIMULAÇÃO NA DOR, FUNÇÃO E PLASTICIDADE CEREBRAL DE INDIVÍDUOS COM OSTEOARTRITE DE JOELHO \\ - o estado da arte.
}

\author{
Cleber Luz Santos*; Diana Oliveira Noronha dos Santos*; Janine Ribeiro Camatti**; Alaí Barbosa \\ Paixão****; Francisco de Brito Xavier ${ }^{* * * *}$; Abrahão Fontes Baptista ${ }^{* * * * *}$

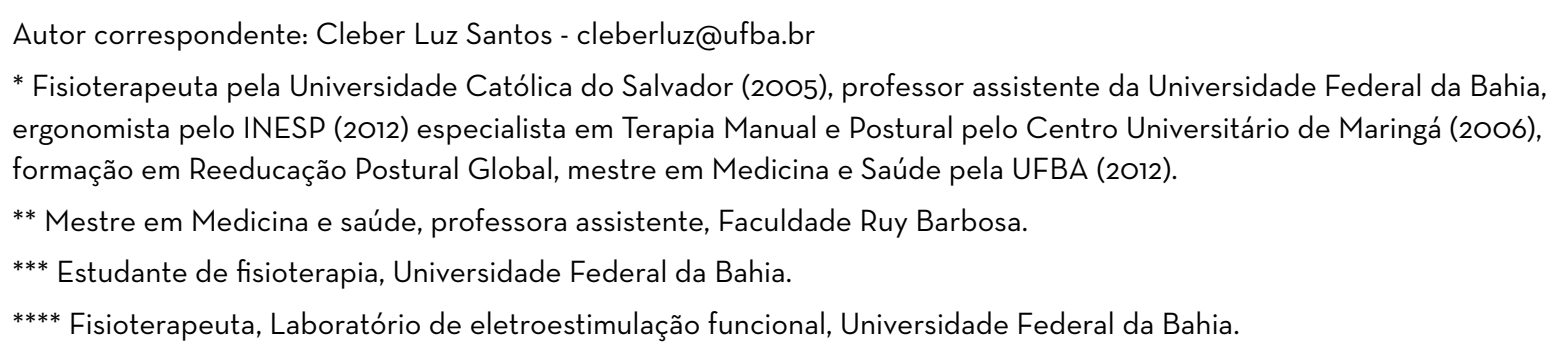

\section{Resumo}

Objetivo: Esta revisão fornece evidências sobre exercício e exercício combinado com a estimulação elétrica em indivíduos com OA do joelho. Método: Uma revisão narrativa foi realizada com termos de estratégias não-farmacológicas, particularmente exercício, estimulação transcraniana por corrente contínua (ETCC) e estimulação elétrica neuromuscular (EEN). As variáveis analisadas foram dor, função, qualidade de vida e excitabilidade cortical. Resultados: Os resultados mostraram que o exercício foi benéfico para melhorar a dor e função em pessoas com OA do joelho. Exercício associados os ETCC pode melhorar os benefícios terapêuticos. As evidências na OA são raras, porém promissoras. Achados semelhantes foram encontrados com o EEN. Estudos sugerem que a combinação é melhor do que isolado exercício. Conclusões: Ambos os estímulos eléctricos pode ser envolvido no exercício com aumento do tamanho do efeito. Isto é devido à melhoria da plasticidade cerebral que ocorre de forma diferente nas duas técnicas. Os efeitos da interação entre os estímulos voluntários (exercício) e exógenos (elétrica) na plasticidade cerebral nos pacientes com OA do joelho requerem maior detalhamento. Informações sobre a intensidade, frequência e duração dos programas de intervenção são divergentes. Revisões sistemáticas com meta-análises podem ser desenvolvidas para identificar a combinação mais efetiva na dor, função e qualidade de vida desses indivíduos.

Palavras-chave: Osteoartrite; Terapia por exercício; Terapia por estimulação elétrica; Plasticidade neuronal; Dor crônica. 


\title{
EXERCISES AND ELECTROSTIMULATION IN PAIN, FUNCTION AND CEREBRAL PLASTICITY OF INDIVIDUALS WITH KNEE OSTEOARTHRITIS
}

- the state of the art $\bullet$

\begin{abstract}
Objective: This review provides evidences about exercise and exercise combined with electrical stimulation in subjects with knee OA. Methods: A narrative review was performed concerning conservative nonpharmacological strategies, particularly exercise, transcranial direct current stimulation (tDCS) and neuromuscular electrical stimulations (NES). The variables analyzed were pain, function, quality of life and cortical excitability. Results: The results have shown what exercises are beneficial for improving pain and function in people with knee OA. Exercise associated the tDCS can improve the benefits from therapeutics. The evidence in OA are rare, however promising. Similar findings were found with NES. Studies suggest what the combination is better than isolated exercise. Conclusions: Electrical stimuli can be involved in the exercise resulting in improved effects. This is due to improved brain plasticity that occurs differently in the two techniques. The effects of the interaction between voluntary stimuli (exercise) and exogenous stimuli (electric) in brain plasticity in the subjects with knee OA require detailing. Information about intensity, frequency and duration of intervention programs are divergent. Systematic reviews with meta-analyses should be developed to identify the most effective combination in pain, function and quality of life to these subjects.
\end{abstract}

Keywords: Osteoarthritis; Exercise therapy; Electrical stimulation therapy; Neuronal plasticity; Chronic pain.

\section{INTRODUÇÃO}

A osteoartrite $(O A)$ de joelho é uma doença com grande impacto na função e na qualidade de vida. Cerca de $18 \%$ das mulheres e $9,6 \%$ dos homens irão desenvolver OA no mundo.(1)Trata-se de uma afecção crônico-degenerativa cujo principal sintoma é a dor crônica (DC). No Brasil, dados epidemiológicos da OA não estão estruturados, mas é possível verificar uma alta prevalência em sujeitos acima de 50 anos. Em Salvador, Bahia, cerca de $11,2 \%$ dos habitantes possuem DC no joelho. ${ }^{(2)}$

Uma estratégia terapêutica consolidada e recomendada por entidades científicas para estes indivíduos são os exercícios físicos. (3) Esta ferramenta terapêutica tem sido amplamente utilizada no con- trole da AO. Terapias complementares como eletroestimulação periférica ou transcraniana quando associadas aos exercícios podem otimizar a reabilitação. Este fato se deve ao aumento da plasticidade cortical que favorece a aquisição do aprendizado motor. Como o córtex dos sujeitos com OA tende a ser menos plástico devido a DC, exercícios isolados podem não ser suficientes para a promover o aprendizado motor. $\mathrm{O}$ resultado esperado nestes casos é a baixa aquisição de benefícios neuromusculares como força, resistência, coordenação motora e equilíbrio. Revisões anteriores demonstraram que os exercícios de força foram parcialmente efetivos na presença da dor. ${ }^{(4)} \mathrm{A}$ plasticidade mal 
adaptada decorrente da dor pode justificar os efeitos terapêuticos abaixo do esperado. ${ }^{(6)} O$ cérebro de um sujeito com dor, tende a ser menos reativo quando comparado aos sujeitos sadios. (7) Portanto, as mudanças plásticas provenientes dos exercícios estarão limitadas ou impedidas na presença da DC. Keysor e Brembs, ${ }^{\left({ }^{8}\right)}$ foram mais enfáticos ao afirmarem que os exercícios são necessários, mas não são suficientes para melhorar a função e prevenir a incapacidade. ${ }^{(8)}$ Diante disto, a combinação de exercícios e eletroestimulação parece trazer mais benefícios quando comparado ao exercício isoladamente.

Novos estudos revelaram que a Estimulação Transcraniana Anódica (ETCCa), aplicada sobre o córtex motor aumenta a reatividade cortical.(9) Efeitos semelhantes no córtex foram observados com a eletroestimulação do QD. (10) Para avaliar a atividade cortical é necessária a Estimulação Magnética Transcraniana (EMT). Este recurso identifica o nível de excitabilidade cortical através de medidas eletrofisiológicas como potencial evocado motor (PEM), Facilitação Intracortical (FIC) e Inibição Intracortical (IIC). O aumento na amplitude do PEM foi observado após aplicação da eletroestimulação e isto significou aumento da excitabilidade corticoespinal.(11) Tanto a estimulação periférica quanto a transcraniana são capazes de aumentar a atividade do córtex motor, contudo, os mecanismos de atuação são distintos.

Atualmente são poucos conhecidos os efeitos da combinação de exercícios e eletroestimulação na plasticidade cortical. A interação entre estas condutas pode potencializar o resultado clínico e otimizar a reabilitação do paciente. Desta forma, é possível vislumbrar um campo de investigação crescente com achados que poderão impactar diretamente na prática fisioterapêutica. Diante disto, esta revisão visa evidenciar os efeitos da associação entre exercícios e eletroestimulação transcraniana ou periférica na dor, função, qualidade de vida e excitabilidade cortical de sujeitos com OA de joelho.

\section{MATERIAIS E MÉTODOS}

Foram realizadas buscas em cinco bases de dados (MEDLINE, Scopus, SPORTDiscus, CINAHL, e PEDro). Os descritores de pesquisa utilizados foram: Osteoartrite de joelho, exercício, estimulação elétrica neuromuscular, estimulação transcraniana por corrente contínua, dor, qualidade de vida e estimulação magnética transcraniana. Esses termos foram usados tanto isoladamente quanto em combinação. Todas as datas e idiomas foram aceitos, sendo inclusos estudos observacionais, ensaios clínicos randomizados e revisões sistemáticas. O período de coleta foi de junho a novembro de 2015 . Os critérios para inclusão foram os seguintes: a) pacientes adultos com diagnóstico de OA de joeIho de acordo com os critérios do American College of Rheumatology; b) grupo intervenção com exercício físico associado ou não a eletroestimulação; c) duração do tratamento com o mínimo de uma sessão; d) desfechos clínicos: dor, função e qualidade de vida; e) Desfechos neurofisiológicos: excitabilidade cortical, bandas de frequências cerebrais e atividade cerebral funcional. Foram excluídos os estudos que: a) Os participantes com OA tivessem realizado cirurgia no joelho; b) Participantes com comorbidades associadas a OA de joelho; c) intervenções que associam drogas ou agentes físicos térmicos. A seleção e a busca foi realizada por um único pesquisador que extraiu os seguintes dados de cada artigo selecionado: autor/ano, características dos participantes, tipo de estudo, intervenção, medidas de desfecho e principais resultados. Estes dados permitiram a discussão dos efeitos de acordo com a intervenção: exercício, exercício associado a estimulação periférica e exercício associado a ETCC.

\section{INTERAÇÃO ENTRE DOR, FUNÇÃO E PLASTICIDADE CORTICAL NA OA DE JOELHO}

A OA é uma das doenças crônico-degenerativa que mais causam disfunção na população. Carac- 
teriza-se pela degradação da cartilagem articular, espessamento do osso subcondral e formação de osteófitos. ${ }^{(3)}$ Quando acomete o joelho resulta em sintomas debilitantes em aproximadamente 10\% das pessoas acima de 55 anos de idade, um quarto destas estão intensamente debilitadas. ${ }^{(12)}$ Desta forma, a doença leva a um grande impacto econômico, gerando altos custos aos sistemas de saúde públi$\mathrm{Ca}^{(13)}$ e gastos indiretos pelos pacientes, tais como adaptações e medicações ${ }^{(14)}$ e custos com perda de emprego ou dificuldades para o trabalho. ${ }^{(15)}$

O reparo natural e remodelação da cartilagem articular são impedidos por cargas mecânicas excessivas nos tecidos articulares. ${ }^{(16)}$ A fonte de estresse mecânico é diversa, mas tem sido postulado que a diminuição do controle postural, (17) disfunção muscular, ${ }^{(18)}$ cargas cumulativas anormais, ${ }^{(19)}$ instabilidade articular e a presença de tecidos anormais dentro da articulação ${ }^{(20)}$ são fatores importantes na gênese da doença.

Os sujeitos com OA de joelho possuem como queixa principal a dor crônica. Estudos retrospectivos revelaram que os sujeitos acometidos tendem a procurar os serviços de saúde quando a dor limita suas atividades diárias. ${ }^{(21)}$ Quando a dor se cronifica ocorre o envolvimento central na $\mathrm{AO}$ denominado de sensibilização central. ${ }^{(22)}$ Neste caso ocorre uma desregulação da percepção álgica que interfere no funcionamento das vias corticoespinais e intracorticais. A sensibilização central na OA tem sido associada à mudanças neuroplásticas na medula espinal e no cérebro. ${ }^{(23)}$ Um estudo recente usando ressonância magnética funcional demonstrou que a DC resultante da OA no joelho não é somente um estado de alteração funcional, mas representa uma "assinatura cerebral" única, indicativa de um processo conhecido como plasticidade mal-adaptativa. Sujeitos com OA de joelho demonstraram volumes diminuídos nos córtices somatosensorial, insular e motor, com o cérebro do indivíduo com OA possuindo mais alterações do que aqueles com dor lombar crônica ou síndrome de dor complexa regional. ${ }^{(6)}$

Embora estes achados reflitam mudanças globais no cérebro devido a DC, dados sobre o córtex motor, em particular, têm fornecido informações chave para guiar técnicas de tratamento conservador. A neuroplasticidade no córtex motor pode ser avaliada através da EMT. Esta técnica não invasiva e indolor usa pulsos magnéticos aplicados no crânio. ${ }^{(24)}$ Quando aplicados no córtex motor primário, pode-se avaliar a excitabilidade da via corticomotora utilizando-se eletromiografia de superfície. Usando a EMT, vários índices de plasticidade corticomotora podem ser medidos, tais como os PEM, IIC e mapeamento da representação de um dado músculo no córtex motor. ${ }^{(24)}$

Uma série de estudos mostraram mudanças nos PEM e IIC em uma variedade de condições dolorosas incluindo dor muscular experimental, ${ }^{(25)}$ dor lombar ${ }^{(26)}$ e fibromialgia. ${ }^{(27)}$ Entretanto apenas dois estudos examinaram o impacto da OA de joelho na plasticidade cortical avaliada com EMT. ${ }^{28,29)}$ Hunt et al., ${ }^{(28)} \mathrm{em}$ um estudo de um único caso, demonstraram que o PEM do músculo reto femoral estava diminuído na presença de OA de joelho, mas que voltaram ao normal após oito semanas de treinamento de força. ${ }^{(28)}$ Kittelson et al., ${ }^{(29)}$ conseguiram evidenciar correlações positivas entre dor e limiar de repouso e também entre dor e FIC. ${ }^{(29)}$

\section{EXERCÍCIOS NA OA DE JOELHO}

O tratamento conservador baseado em exercícios terapêuticos pode melhorar função e a Qualidade de Vida (QV) de sujeitos com AO de joelho. ${ }^{\left({ }^{\circ}\right)}$ Exercícios físicos supervisionados de baixa intensidade são considerados padrão-ouro para dor e disfunção na AO. ${ }^{(31}{ }^{2014}$ Embora os benefícios clínicos sejam evidenciados em diversos estudos, ${ }^{(18,34)}$ os parâmetros de intensidade, duração e frequência são difusos.

Revisões sistemáticas demonstraram que os exercícios de força foram benéficos apenas no curto prazo. ${ }^{(31) 2014}$ Numa revisão sobre treinamento de força e equilíbrio em idosos com OA de joelho, os resultados indicaram baixo tamanho de efeito e elevada variação na dosagem dos exercícios. ${ }^{(33)}$ Exercícios terapêuticos em solo tiveram um efeito benéfico somente a curto prazo em termos de re- 
dução da dor e melhora na função física de pessoas com OA de joelho, com pequena magnitude de efeito do tratamento. ${ }^{\left({ }^{2}\right)}$ Iwamoto et al., (34) numa revisão da literatura destacaram que os exercícios de força apresentaram efeitos benéficos nos estágios leve e moderado da OA de joelho.

As adaptações neuroplásticas decorrentes da DC são mais evidentes nos quadros crônicos de OA e este fato pode dificultar o controle da dor e da função. Através da ressonância magnética funcional foi possível observar que os sujeitos com DC secundário à $O A$ de quadril apresentaram uma atrofia talâmica e que a melhora somente ocorreu após a artroplastia total desta articulação. ${ }^{(35)}$ As inferências somatosensoriais de dor foram decisivas para modificar o funcionamento talâmico. A interação talamocortical é importante no controle dos movimentos e da percepção somatosensorial. Quando há um desequilíbrio entre estas estruturas, ocorre a Disritmia Talamocortical (DTC). A DTC pode ser evidenciada pela redução do ritmo beta na Eletroencefalografia Quantitativa (EEGq). ${ }^{(36)} \mathrm{Em}$ sujeitos com fibromialgia a DTC dificultou o controle da dor nestes sujeitos. ${ }^{(37)}$ Independente da doença, a DC é capaz de provocar a DTC e afetar a plasticidade cortical. ${ }^{\left({ }^{8}\right)}$ Não foram encontrados estudos que caracterizassem a DTC na OA.

Os exercícios de força podem ser menos efetivos na presença da DTC. Isto se deve a dificuldade de aquisição de valências clínicas associadas ao aprendizado motor como força muscular, resistência, coordenação motora, equilíbrio. Os efeitos clínicos dos exercícios foram investigados por diferentes autores com achados benéficos consistentes. ${ }^{(32,34,39)} A$ variedade de modalidades terapêuticas com parâmetros de intensidade, frequência e duração inconstantes dificulta a comparação entre estes estudos. Exercícios de baixa ou alta intensidade parecem atuar de modo semelhante no controle da dor, função e capacidade aeróbica destes sujeitos e ambos os exercícios demonstraram ser mais efetivos que não praticar nenhuma atividade física. ${ }^{(40)}$ Este fato pode ser justificado pela baixa qualidade de alguns estudos de acordo com os critérios do CONSORT. ${ }^{(41)}$
Segundo Bennell et al, ${ }^{(42)}$ os exercícios aeróbicos e de fortalecimento são complementares e beneficiam os sujeitos de forma distinta. O exercício resistido demonstrou ser mais efetivo no curto prazo para redução da dor quando comparado com exercícios aeróbicos. ${ }^{(39)}$ Em contrapartida, o exercício aeróbico proporciona mais ganhos funcionais no longo prazo. Um achado importante nesta revisão foi que a prática de qualquer exercício físico foi melhor do que não realizar nenhuma atividade. Esta informação fomenta uma discussão sobre o papel do sedentarismo na gênese da $A O$ de joelho. Contudo, uma nova revisão deve ser implementada para analisar esta relação.

Outra evidência que merece destaque nesta revisão é o papel do exercício domiciliar na reabilitação dos sujeitos com $A O$ de joelho. As evidências encontradas apontam uma tendência a prescrição deste tipo de exercício. Uma revisão sistemática com metanálise de 13 ensaios clínicos randomizados conseguiu evidenciar que o treino aeróbio através de caminhada e o fortalecimento do quadríceps foram capazes de reduzir a intensidade da dor com tamanho de efeito de 0,52 e 0,39 respectivamente. (39) A prescrição de exercícios domiciliares tem sido uma estratégia válida e recomendada na reabilitação de sujeitos com $O A$ de joelho. $A$ adesão dos sujeitos deve ser monitorada pelo profissional com visitas periódicas. Quando não ocorre esta supervisão, os exercícios domiciliares podem não ser efeitivos como demonstrado por Fransen et al, ${ }^{(43)}$ Esta prática parece atender melhor a demanda dos pacientes, além de torná-lo pró-ativo em sua reabilitação. Esta abordagem clínica se adequa a visão biopsicossocial proposta pela classificação internacional de funcionalidade.

\section{ELETROESTIMULAÇÃO NA OA DE JOELHO}

A falta de um ganho a longo prazo pode ser explicada pela dificuldade dos exercícios em reverter as mudanças plásticas no córtex motor resultantes do quadro doloroso. Modular a atividade cortical para 
que o pool de neurônios motores se torne mais reativo aos estímulos provenientes dos exercícios pode potencializar o tamanho de efeito dos exercícios. Isto pode ser possível através da aplicação de técnicas de eletroestimulação. A plasticidade cortical pode ser modulada tanto por meios não-invasivos que atuam diretamente no sistema nervosa central como com técnicas periféricas, ${ }^{(10,)}$ ou sua interação. ${ }^{(25)}$

\section{a) Estimulação Transcraniana e exercícios}

A ETCC é uma nova técnica de neuromodulação com um potencial para atingir as mudanças mal-adaptativas consequentes de quadros de dor crônica. Esta é uma técnica simples, que envolve a aplicação de uma corrente elétrica de baixa intensidade através do crânio. ${ }^{(44)}$ Considera-se que esta corrente elétrica pode modificar o estado basal da atividade neuronal no córtex motor, levando a mudanças na excitabilidade cortical. Ensaios clínicos usando ETCC para o tratamento da dor têm demonstrado efeitos moderados, embora existam variabilidades nos resultados. A modulação através do ânodo no córtex motor primário aumenta o potencial de membrana e leva a efeitos analgésicos. (45) Apesar dos seus efeitos promissores como uma ferramenta terapêutica, poucos estudos associam a ETCC com outras técnicas direcionadas a função muscular, como exercícios. O baixo lastro científico pode dificultar a construção de uma revisão sistemática, mas ampliou o campo de investigação de estratégias conservadoras mais efetivas na OA de joelho.

Esta associação entre ETCC e exercícios para AO de joelho é recente e foram encontrados apenas protocolos de intervenção para esta população. ${ }^{(46)}$ Resultados específicos para $\mathrm{AO}$ de joelho ainda não foram publicados, mas dados envolvendo lesados medulares ${ }^{(47)}$ e acidente vascular cerebral ${ }^{(48)}$ revelaram benefícios com esta combinação. A disfunção de mecanismos centrais e periféricos decorrentes da dor parecem ser mais influenciados pela interação entre exercícios (atividade voluntária) e ele- troestimulação (estímulo exógeno). Para o Sistema Nervoso Central (SNC), os exercícios geram inputs sensoriais (proprioceptores e mecaniceptores) que modificam o controle muscular (coordenação motora e força). Uma repercussão clínica relevante neste mecanismo é a analgesia induzida pelo exercício. Este fato se deve a liberação de opióides nas vias descendentes de controle da dor. ${ }^{(49)}$

O processamento central destes inputs pode estar prejudicado se as membranas pós sinápticas estiverem hipoativas ou até inativas. A atividade neuronal reduzida pode ser revertida com técnicas diretas no SNC como a ETCC. Isto significa que a ETCC é capaz de melhorar a receptividade do cérebro aos estímulos provenientes dos exercícios. Esta base teórica justifica o uso combinado das duas técnicas, sendo aplicado primeiramente a ETCC.

\section{b) Estimulação periférica e exercícios}

A Estimulação Periférica (EP) também é capaz de modificar o funcionamento cerebral.(10) Esta afirmação refuta o paradigma de que as correntes periféricas não são capazes de interferir na plasticidade cerebral. Uma modalidade amplamente utilizada é a estimulação elétrica nervosa transcutânea (TENS) que na $\mathrm{AO}$ de joelho pode ser aplicado diretamente no joelho ou na musculatura da coxa. Mudanças plásticas foram observadas quando esta corrente elétrica foi aplicada na coxa de sujeitos saudáveis. (11) Os efeitos estavam diretamente ligados aos parâmetros da corrente.

Chipchase et al,(10) na sua revisão sistemática demonstrou que a intensidade da corrente é decisiva neste processo, mas a frequência ainda necessita de mais estudos e o formato do pulso parece não influenciar na modulação cortical. Intensidades altas no limiar motor tendem a aumentar a excitabilidade. Mas se a intensidade atingir o limiar nocivo, ocorrerá uma redução da excitabilidade cortical. Intensidade sensorial tende a inibir as vias corticoespinais. A frequência mantida a zohz demonstrou maior capacidade de aumentar a excitabilidade 
cortical, contudo, frequência de 10ohz associada ao limiar sensitivo demonstraram efeito inibitório na via corticoespinal. Esta informação possibilitar inferir que fazer TENS sensorial com alta frequência antes de um programa de exercício parece diminuir a responsividade do córtex motor aos exercícios. O contrário parece ser verdadeiro ao realizar TENS motor à zohz parece aumentar a responsividade aos exercícios.

A prescrição correta dos parâmetros da estimulação periférica tem efeito direto nos resultados. A estimulação motora que gere contração muscular é a melhor indicação para otimizar os resultados dos exercícios. Elboim-Gabyzon et al, (50) demonstraram que a estimulação motora do quadríceps melhorou a efetividade de um programa de exercícios para $\mathrm{AO}$ de joelho. Este resultado foi obtido com estimulação no limiar motor a $75 \mathrm{~Hz}$ e $250 \mu \mathrm{s}$. Apesar desta evidência, uma revisão sistemática concluiu que os achados de estimulação periférica e exercício na $A O$ de joelho são ambíguos. A explicação para este fato se deve a heterogeneidade. Baseado neste achado, a combinação de estimulação periférica motora e exercícios parece ser mais benéfico na dor e na função e que isto está diretamente relacionado à plasticidade cerebral.

\section{CONCLUSÃO}

Em resumo, exercícios terapêuticos para OA de joelho demonstraram efetividade limitada e baixos tamanhos de efeito. Uma série de evidências sugerem que a dor e disfunção associada a OA são resultados de mudanças periféricas, mas também centrais. Um tratamento que tenha como alvo a plasticidade mal-adaptativa no córtex motor pode potencializar os efeitos de exercícios e de técnicas de eletroestimulação periférica, levando a resultados clínicos mais duradouros. Desta forma, a aplicação de eletroestimulação transcraniana ou periférica, mas não a combinação entre ambas antes dos exercícios, pode favorecer os efeitos terapêuticos na dor e na função em sujeitos com AO.
A divergência no detalhamento dos parâmetros de intensidade, frequência e duração dos programas de intervenção demonstram a necessidade de uma revisão sistemática que identifique a combinação que seja mais efetiva no controle da dor, função e qualidade de vida dos sujeitos com OA de joelho.

\section{REFERÊNCIAS}

1. March LM, Bagga H. Epidemiology of osteoarthritis in Australia. Med J Aust [Internet]. 2004 Mar 1 [cited 2015 May 8];180(5 Suppl):S6-1O. Available from: http://www.ncbi. nlm.nih.gov/pubmed/14984356

2. Sá KN, Pereira $C$ de M, Souza RC, Baptista AF, Lessa I. Knee pain prevalence and associated factors in a Brazilian population study. Pain med [Internet]. 2011 Mar [cited $2015 \mathrm{Dec}$ 16];12(3):394-4O2. Available from: http://www. ncbi.nlm.nih.gov/pubmed/21332935

3. Jordan KM, Arden NK, Doherty M, Bannwarth B, Bijlsma JWJ, Dieppe P, et al. EULAR Recommendations 2003: an evidence based approach to the management of knee osteoarthritis: Report of a Task Force of the Standing Committee for International Clinical Studies Including Therapeutic Trials (ESCISIT). Ann Rheum Dis [Internet]. 2003 Dec [cited 2015 Dec 16];62(12):1145-55. Available from: http://www.pubmedcentral.nih.gov/articlerender. fcgi?artid=1754382\&tool=pmcentrez\&rendertyp $\mathrm{e}=$ abstract

4. Fransen M, McConnell S. Land-based exercise for osteoarthritis of the knee: a metaanalysis of randomized controlled trials. J Rheumatol [Internet]. 2009 Jun [cited 2014 Nov 30];36(6):1109-17. Available from: http://www. ncbi.nlm.nih.gov/pubmed/19447940

5. Jansen MJ, Viechtbauer W, Lenssen AF, Hendriks EJM, de Bie RA. Strength training alone, exercise therapy alone, and exercise therapy with passive manual mobilisation each reduce pain and disability in people with knee osteoarthritis: a systematic review. J Physiother [Internet]. 2011 Jan [cited 2015 Nov 26];57(1):11- 
2O. Available from: http://www.ncbi.nlm.nih.gov/ pubmed/21402325

6. Baliki MN, Schnitzer TJ, Baver WR, Apkarian AV. Brain morphological signatures for chronic pain. PloS one [Internet]. 2011 Jan [cited 2014 Oct 17];6(10):e26010. Available from: http://www. pubmedcentral.nih.gov/articlerender.fcgi?artid= 3192794\&tool=pmcentrez\&rendertype=abstract

7. Labus JS, Van Horn JD, Gupta A, Alaverdyan M, Torgerson C, Ashe-McNalley C, et al. Multivariate morphological brain signatures predict patients with chronic abdominal pain from healthy control subjects. Pain [Internet]. 2015 Aug [cited 2015 Dec 16];156(8):1545-54. Available from: http://www.ncbi.nlm.nih.gov/ pubmed/25906347

8. Keysor JJ, Brembs A. Exercise: necessary but not sufficient for improving function and preventing disability? Curr Opin Rheumatol [Internet]. 2011 Mar [cited 2015 Dec 22];23(2):211-8. Available from: http://www.ncbi.nlm.nih.gov/ pubmed/21252681

9. Devanathan D, Madhavan S. Effects of anodal tDCS of the lower limb MI on ankle reaction time in young adults. Exp Brain Res [Internet]. 2015 Oct 20 [cited 2015 Dec 16]; Available from: http://www.ncbi.nlm.nih.gov/ pubmed/26487179

10. Chipchase LS, Schabrun SM, Hodges PW. Peripheral electrical stimulation to induce cortical plasticity: a systematic review of stimulus parameters. Clin Neurophysiol [Internet]. 2011 Mar [cited 2014 Nov 19];122(3):456-63. Available from: http:// www.sciencedirect.com/science/article/pii/ S1388245710006103

11. Chipchase LS, Schabrun SM, Hodges PW. Corticospinal excitability is dependent on the parameters of peripheral electric stimulation: a preliminary study. Arch Phys Med Rehabil [Internet]. 2011 Sep [cited 2014 Dec 18];92(9):1423-30. Available from: http://www. ncbi.nlm.nih.gov/pubmed/21620374

12. Plotnikoff R, Karunamuni N, Lytvyak E, Penfold C, Schopflocher D, Imayama I, et al. Osteoarthritis prevalence and modifiable factors: a population study. BMC public health [Internet]. 2015 Jan [cited 2015 Dec 16];15:1195.
Available from: http://www.pubmedcentral.nih. gov/articlerender.fcgi?artid $=4666016 \&$ tool=pmc entrez\&rendertype=abstract

13. Elders MJ. The increasing impact of arthritis on public health. J Rheumatol [Internet]. 2000 Oct [cited 2015 Dec 15];60:6-8. Available from: http://www.ncbi.nlm.nih.gov/pubmed/11032095

14. MacLean $\mathrm{CH}$, Knight K, Paulus H, Brook RH, Shekelle PG. Costs attributable to osteoarthritis. J Rheumatol [Internet]. 1998 Nov [cited 2015 Dec 16];25(11):2213-8. Available from: http:// www.ncbi.nlm.nih.gov/pubmed/9818666

15. Gupta S, Hawker GA, Laporte A, Croxford R, Coyte PC. The economic burden of disabling hip and knee osteoarthritis (OA) from the perspective of individuals living with this condition. Rheumatology (Oxford) [Internet]. 2005 Dec [cited 2015 Dec 16];44(12):1531-7. Available from: http://www.ncbi.nlm.nih.gov/ pubmed/16091394

16. Brandt KD, Dieppe P, Radin E. Etiopathogenesis of osteoarthritis. Med Clin North Am [Internet]. 2009 Jan [cited 2014 Dec 18];93(1):1-24, xv. Available from: http://www.ncbi.nlm.nih.gov/ pubmed/19059018

17. Sanchez-Ramirez DC, van der Leeden M, Knol DL, van der Esch M, Roorda LD, Verschueren $\mathrm{S}$, et al. Association of postural control with muscle strength, proprioception, self-reported knee instability and activity limitations in patients with knee osteoarthritis. J Rehabil Med [Internet]. 2013 Feb [cited 2015 Dec 16];45(2):192-7. Available from: http://www.ncbi. nlm.nih.gov/pubmed/23223845

18. Knoop J, van der Leeden M, van der Esch M, Thorstensson CA, Gerritsen M, Voorneman $\mathrm{RE}$, et al. Association of lower muscle strength with self-reported knee instability in osteoarthritis of the knee: results from the Amsterdam Osteoarthritis cohort. Arthritis Care Res [Internet]. 2012 Jan [cited 2015 Dec 16];64(1):38-45. Available from: http://www.ncbi. nlm.nih.gov/pubmed/22213723

19. Block JA, Shakoor N. Lower limb osteoarthritis: biomechanical alterations and implications for therapy. Curr Opin Rheumatol [Internet]. 2010 Sep [cited 2014 Dec 13];22(5):544-50. 
Available from: http://www.ncbi.nlm.nih.gov/ pubmed/20592605

2O. Lane NE, Brandt K, Hawker G, Peeva E, Schreyer E, Tsuji W, et al. OARSI-FDA initiative: defining the disease state of osteoarthritis. Osteoarthr Cartil [Internet]. 2011 May [cited 2015 Dec 16];19(5):478-82. Available from: http://www.ncbi.nlm.nih.gov/pubmed/21396464

21. Marshall DA, Vanderby S, Barnabe C, MacDonald K V, Maxwell C, Mosher D, et al. Estimating the Burden of Osteoarthritis to Plan for the Future. Arthritis Care Res [Internet]. 2015 Oct [cited 2015 Dec 16];67(10):1379-86. Available from: http://www.ncbi.nlm.nih.gov/ pubmed/25939659

22. Baños-Díez JE, Navarro-Acebes X. [Current aspects of nociceptive transmission: peripheral mechanisms and spinal modulation]. Rev Neurol [Internet]. $1996 \mathrm{Jul}$ [cited $2015 \mathrm{Dec}$ 16];24(131):769-78. Available from: http://www. ncbi.nlm.nih.gov/pubmed/8681187

23. Howard MA, Sanders D, Krause K, O'Muircheartaigh J, Fotopoulou A, Zelaya $\mathrm{F}$, et al. Alterations in resting-state regional cerebral blood flow demonstrate ongoing pain in osteoarthritis: An arterial spin-labeled magnetic resonance imaging study. Arthritis Rheum [Internet]. 2012 Dec [cited 2015 May 8];64(12):3936-46. Available from: http://www. ncbi.nlm.nih.gov/pubmed/22933378

24. Hallett M. Transcranial magnetic stimulation: a primer. Neuron [Internet]. $2007 \mathrm{Jul} 19$ [cited 2015 Jun 23];55(2):187-99. Available from: http:// www.ncbi.nlm.nih.gov/pubmed/17640522

25. Schabrun SM, Hodges PW. Muscle pain differentially modulates short interval intracortical inhibition and intracortical facilitation in primary motor cortex. J Pain [Internet]. 2012 Feb [cited 2015 Jun 11];13(2):18794. Available from: http://www.ncbi.nlm.nih.gov/ pubmed/22227117

26. Tsao H, Galea MP, Hodges PW. Reorganization of the motor cortex is associated with postural control deficits in recurrent low back pain. Brain [Internet]. 2008 Aug [cited 2015 Dec 16];131(Pt 8):2161-71. Available from: http://www.ncbi.nlm. nih.gov/pubmed/18669505
27. Mhalla A, de Andrade DC, Baudic S, Perrot S, Bouhassira D. Alteration of cortical excitability in patients with fibromyalgia. Pain [Internet]. 2010 Jun [cited 2015 Feb 22];149(3):495-500. Available from: http://www.ncbi.nlm.nih.gov/ pubmed/2O356675

28. Hunt MA, Zabukovec JR, Peters S, Pollock CL, Linsdell MA, Boyd LA. Reduced quadriceps motor-evoked potentials in an individual with unilateral knee osteoarthritis: a case report. Case Rep Rheumatol [Internet]. 2011 Jan [cited 2015 Jun 11];2011:53742O. Available from: http:// www.pubmedcentral.nih.gov/articlerender.fcgi? artid $=3420629 \&$ tool=pmcentrez\&rendertype $=a$ bstract

29. Kittelson AJ, Thomas AC, Kluger BM, StevensLapsley JE. Corticospinal and intracortical excitability of the quadriceps in patients with knee osteoarthritis. Exp Brain Res [Internet]. 2014 Dec [cited 2015 Dec 23];232(12):3991-9. Available from: http://www.pubmedcentral.nih. gov/articlerender.fcgi?artid=4404306\&tool=pm centrez\&rendertype=abstract

30. Tanaka R, Ozawa J, Kito N, Moriyama H. Does exercise therapy improve the healthrelated quality of life of people with knee osteoarthritis? A systematic review and metaanalysis of randomized controlled trials. J Phys Ther Sci [Internet]. 2015 Oct [cited 2015 Dec 9];27(10):3309-14. Available from: http://www. pubmedcentral.nih.gov/articlerender.fcgi?artid= 4668190\&tool=pmcentrez\&rendertype=abstract

31. Regnaux J-P, Lefevre-Colau M-M, Trinquart L, Nguyen C, Boutron I, Brosseau L, et al. High-intensity versus low-intensity physical activity or exercise in people with hip or knee osteoarthritis. Cochrane Database Syst Rev [Internet]. 2015 Jan [cited 2015 Nov 13];1O:CDO1O2O3. Available from: http://www. ncbi.nlm.nih.gov/pubmed/26513223

32. Fransen M, McConnell S. Exercise for osteoarthritis of the knee. Cochrane Database Syst Rev [Internet]. 2008 Jan [cited 2014 Dec 1];(4):CDOO4376. Available from: http://www. ncbi.nlm.nih.gov/pubmed/18843657

33. Mat S, Tan MP, Kamaruzzaman SB, Ng CT. Physical therapies for improving balance and reducing falls risk in osteoarthritis of the knee: a systematic review. Age ageing [Internet]. 
2015 Jan [cited 2015 Dec 3];44(1):16-24.

Available from: http://www.ncbi.nlm.nih.gov/ pubmed/25149678

34. Iwamoto J, Sato Y, Takeda T, Matsumoto H. Effectiveness of exercise for osteoarthritis of the knee: A review of the literature. World J Orthop [Internet]. 2011 May 18 [cited 2015 Dec 17];2(5):37-42. Available from: http://www. pubmedcentral.nih.gov/articlerender.fcgi?artid $=3302040 \&$ tool $=$ pmcentrez\&rendertype $=$ abst ract

35. Gwilym SE, Filippini N, Douaud G, Carr AJ, Tracey I. Thalamic atrophy associated with painful osteoarthritis of the hip is reversible after arthroplasty: a longitudinal voxel-based morphometric study. Arthritis Rheum [Internet]. 2010 Oct [cited 2015 Dec 16];62(10):2930-4O. Available from: http://www.ncbi.nlm.nih.gov/ pubmed/20518076

36. Schmidt S, Naranjo JR, Brenneisen C, Gundlach J, Schultz C, Kaube H, et al. Pain ratings, psychological functioning and quantitative EEG in a controlled study of chronic back pain patients. PloS one [Internet]. 2012 Jan [cited 2015 Dec 22];7(3):e31138. Available from: http:// www.pubmedcentral.nih.gov/articlerender.fcgi? artid $=3303776 \&$ tool=pmcentrez\&rendertype $=a$ bstract

37. Saavedra L, Mendonca M, Fregni F. Role of the primary motor cortex in the maintenance and treatment of pain in fibromyalgia. Med hypot [Internet]. 2014 Sep [cited 2015 Dec 22];83(3):332-6. Available from: http://www.ncbi. nlm.nih.gov/pubmed/24992875

38. Jones EG. Thalamocortical dysrhythmia and chronic pain. Pain [Internet]. $2010 \mathrm{Jul}$ [cited 2015 Dec 23];15O(1):4-5. Available from: http:// www.ncbi.nlm.nih.gov/pubmed/20395046

39. Roddy E, Zhang W, Doherty M. Aerobic walking or strengthening exercise for osteoarthritis of the knee? A systematic review. Ann Rheum Dis [Internet]. 2005 Apr [cited 2015 Nov 30];64(4):544-8. Available from: http://www. pubmedcentral.nih.gov/articlerender.fcgi?artid=1 755453\&tool=pmcentrez\&rendertype=abstract

4O. Brosseau L, MacLeay L, Robinson V, Wells G, Tugwell P. Intensity of exercise for the treatment of osteoarthritis. Cochrane Database Syst
Rev [Internet]. 2003 Jan [cited 2015 Dec 17];(2):CDOO4259. Available from: http://www. ncbi.nlm.nih.gov/pubmed/12804510

41. Altman DG, Schulz KF, Moher D, Egger M, Davidoff F, Elbourne D, et al. The revised CONSORT statement for reporting randomized trials: explanation and elaboration. Ann Intern Med [Internet]. 2001 Apr 17 [cited 2015 Sep 26];134(8):663-94. Available from: http://www. ncbi.nlm.nih.gov/pubmed/11304107

42. Bennell K, Hinman R. Exercise as a treatment for osteoarthritis. Curr Opin Rheumatol [Internet]. 2005 Sep [cited 2015 Dec 29];17(5):634-4O. Available from: http://www.ncbi.nlm.nih.gov/ pubmed/16093845

43. Fransen M, McConnell S, Bell M. Therapeutic exercise for people with osteoarthritis of the hip or knee. A systematic review. J Rheumatol [Internet]. 2002 Aug [cited 2015 Dec 17];29(8):1737-45. Available from: http://www. ncbi.nlm.nih.gov/pubmed/12180738

44. Schabrun SM, Chipchase LS. Priming the brain to learn: the future of therapy? Man Ther [Internet]. 2012 Apr [cited 2015 Dec 16];17(2):184-6. Available from: http://www.ncbi. nlm.nih.gov/pubmed/22197081

45. Marlow NM, Bonilha HS, Short EB. Efficacy of transcranial direct current stimulation and repetitive transcranial magnetic stimulation for treating fibromyalgia syndrome: a systematic review. Pain Pract [Internet]. 2013 Feb [cited 2015 Dec 16];13(2):131-45. Available from: http:// www.ncbi.nlm.nih.gov/pubmed/22631436

46. Chang W-J, Bennell KL, Hodges PW, Hinman RS, Liston MB, Schabrun SM. Combined exercise and transcranial direct current stimulation intervention for knee osteoarthritis: protocol for a pilot randomised controlled trial. BMJ open [Internet]. 2015 Jan [cited 2015 Dec 24];5(8):eOO8482. Available from: http://www. pubmedcentral.nih.gov/articlerender.fcgi?artid $=4550738 \&$ tool $=$ pmcentrez $\&$ rendertype $=a b s t r$ act

47. Silva FTG, Rêgo JTP, Raulino FR, Silva MR, Reynaud F, Egito EST, et al. Transcranial direct current stimulation on the autonomic modulation and exercise time in individuals with spinal cord injury. A case report. Auton Neurosci 
[Internet]. 2015 Dec [cited 2015 Dec 10];193:1525. Available from: http://www.ncbi.nlm.nih.gov/ pubmed/26329874

48. Park SD, Kim JY, Song HS. Effect of application of transcranial direct current stimulation during task-related training on gait ability of patients with stroke. J Phys Ther Sci [Internet]. 2015 Mar [cited 2015 Dec 24];27(3):623-5. Available from: http://www.pubmedcentral.nih.gov/articlerender. fcgi?artid=4395678\&tool=pmcentrez\&rendertyp e=abstract

49. Millan MJ. Descending control of pain. Prog Neurobiol [Internet]. 2002 Apr [cited 2015 Sep
2];66(6):355-474. Available from: http://www. ncbi.nlm.nih.gov/pubmed/12034378

50. Elboim-Gabyzon M, Rozen N, Laufer Y. Does neuromuscular electrical stimulation enhance the effectiveness of an exercise programme in subjects with knee osteoarthritis? A randomized controlled trial. Clin Rehabil [Internet]. 2013 Mar [cited 2015 Nov 23];27(3):246-57. Available from: http://www.ncbi.nlm.nih.gov/ pubmed/22952305 